\title{
LA TRADUCCIÓN LITERARIA Y LA BRECHA DE PARALAJE. REFLEXIONES A PARTIR DE UN CUESTIONARIO PILOTO ${ }^{1}$
}

\author{
Fruela Fernández \\ Universidad de Granada
}

\section{Resumen}

La investigación social se ha movido históricamente entre oposiciones conceptuales que el construccionismo sociológico intenta superar. Mediante el análisis de las respuestas y los problemas planteados por un cuestionario piloto dirigido a traductores de literatura, se tratan una serie de planteamientos aplicables a la investigación sociológica de la traducción literaria. Se hace especial hincapié en la indefinición como grupo de los traductores literarios y se plantea la utilidad del concepto "brecha de paralaje" (Slavoj Žižek) como método de análisis interdisciplinar y dialéctico.

\begin{abstract}
Historically, social research has alternated between conceptual opposites that social constructionism tries to reconcile. By analyzing the issues raised and responses given to a pilot questionnaire completed by literary translators, this article discusses approaches that could be applied to sociological research in literary translation. Special emphasis is placed on the undefined nature of literary translators as a group and on the usefulness of the "parallax gap" concept (Slavoj Žižek) as a dialectical, interdisciplinary method of analysis.
\end{abstract}

\section{Palabras clave}

Traducción literaria. Traducción profesional. Cuestionarios. Brecha de paralaje. Sociología de la traducción.

\section{Keywords}

Literary translation. Professional translation. Questionnaires. Parallax Gap. Sociology of translation.

1. El autor de este artículo es beneficiario de una beca de investigación del programa F.P.U. del Ministerio de Educación y Ciencia (referencia AP2006-02234). 


\section{Introducción: las oposiciones conceptuales en investigación social}

Tradicionalmente las investigaciones sociales se han movido entre pares de conceptos opuestos que delimitan su ámbito de trabajo y condicionan su enfoque: material vs. ideal, objetivo vs. subjetivo, colectivo vs. individual ( $c f$. Rubinstein 1981: 10-88; Corcuff 1995: 11-22). Aunque la dependencia respecto a estos paired concepts (Bendix \& Berger 1959) haya supuesto una traba para la comprensión de fenómenos tan complejos como los sociales, la investigación en este campo no deja de estar asediada, de manera continua, por presencias sutiles de estas dicotomías.

Una de las discusiones más complejas y de influencia más persistente sigue siendo la que enfrenta a "actores" y a "observadores", cuyo paradigma clásico es la distinción entre los participantes en una determinada situación social y los investigadores que los analizan; oposición que se ha hecho habitual bajo la denominación antropológica emics vs. etics, planteada por Pike (1954) y reinterpretada posteriormente por Marvin Harris (1964, 1979). Si bien la pareja emics-etics sigue siendo controvertida, ya que plantea a su vez nuevos interrogantes ( $c f$. el debate desarrollado en Headland, Pike \& Harris 1990), resulta evidente que alude a una preocupación básica de la investigación social: los problemas planteados por la diferencia de perspectivas sobre el "objeto" sociológico que poseen actores e investigadores y las posibles inexactitudes o confusiones derivadas de tal diferencia. El planteamiento ya aparece, por ejemplo, con Durkheim, quien se decantaba en este debate siguiendo una filosofía materialista de la historia: "Consideramos fecunda la idea de que la vida social no debe explicarse por la concepción que se forman quienes participan en ella, sino por causas profundas que escapan a la conciencia [...]" (Durkheim 1897: 250; traducción propia). Justamente de Durkheim y de Mauss proviene el concepto de "ruptura epistemológica" reivindicado por Pierre Bourdieu: en las ciencias humanas, el investigador ha de mantener una vigilancia constante sobre sus métodos, ya que en estas disciplinas "la separación entre la opinión común y el discurso científico es más imprecisa que en otros casos" (Bourdieu, Chamboredon \& Passeron 1975: 27). Del lado contrario se situarían aquellos investigadores y pensadores que no desconfían 
de la opinión de los participantes, sino que se centran precisamente en su incitación; desde esta perspectiva, el intelectual o investigador ya no sería el descubridor de algo que "se escapa a la conciencia", sino el encargado de luchar contra "el sistema de poder" que intercepta el discurso de los participantes y evita que se oiga:

[Tradicionalmente] el intelectual decía la verdad a los que todavía no la veían y en nombre de los que no podían decirla: conciencia y elocuencia.

Ahora bien, los intelectuales han descubierto, después de las recientes luchas [de mayo del 68], que las masas no los necesitan para saber; ellas saben perfectamente, claramente, mucho mejor que ellos; y además lo dicen muy bien. (Foucault 1972: 25)

Aunque probablemente estas dicotomías nunca llegan a ser radicales en ningún sistema de investigación moderno, las distintas herencias e influencias intelectuales, además de la presencia sutil de conceptos asumidos, requieren una vigilancia constante frente a estas separaciones que pueden oscurecer el planteamiento de trabajo. Un planteamiento constructivista, como el que guía esta investigación, parte, por tanto, de la premisa de que es imprescindible "establecer vías de comunicación entre el punto de vista exterior del observador y las formas en que los actores perciben y viven lo que hacen mientras actúan" (Corcuff 1995: 15); planteamiento que exige, asimismo, "reflexividad sociológica" ( $c f$. Bourdieu 2001: 167-220), es decir, un análisis del estudioso acerca de su propia relación con el objeto estudiado.

En este sentido, esta investigación también reivindica la noción de "brecha de paralaje" desarrollada por Slavoj Žižek (2005). Técnicamente, se denomina paralaje al aparente desplazamiento de un objeto (astronómico) a causa de un cambio en la posición desde la que se observa; aplicada a la investigación política y social, la paralaje permite hacer referencia al cambio en la percepción de los distintos hechos que constituyen la "realidad" colectiva. Ahora bien, Žižek -en paralelismo con Katarani (2001)- introduce un matiz importante en esta reaplicación: la diferencia que muestra la paralaje social no se puede considerar subjetiva y contingente, sino epistemológica y, por tanto, ontológica, ya que el cambio en la perspectiva de investigación transforma el objeto; desde una línea distinta, Bachelard ya había afirmado que el hecho científico se construye. De acuerdo con el planteamiento de Žižek, el análisis crítico más productivo sería, en suma, el dialéctico, aquel que se situase justamente en la "brecha" entre las distintas paralajes; es decir, el análisis que no plantee las distintas oposiciones entre perspectivas (como la dicotomía participante-observador), sino que trabaje a partir del intersticio entre ellas (cf. Žižek 2005: 25-29). 


\section{Análisis de un cuestionario piloto: el problema como posibilidad}

\subsection{Propósitos de la investigación y aspectos metodológicos}

Con el objetivo de establecer precisamente las "vías de comunicación" entre el investigador y los actores que puedan definir la "brecha" de trabajo y aportar nuevas perspectivas a una investigación en curso sobre la traducción literaria en España (Fernández 2007, 2008 y en preparación), se confeccionó un cuestionario dirigido a traductores de literatura; el cuestionario debía servir, en cierto modo, como medio de contraste entre los planteamientos de investigación desarrollados hasta entonces y las opiniones de los participantes acerca del ámbito profesional-cultural en el que desarrollaban su actividad. Dicho cuestionario fue pretestado entre mayo y junio de 2008; aunque por razones de espacio no sea posible incluir el cuestionario piloto en este artículo, se detallarán brevemente algunas cuestiones metodológicas que guiaron el proceso, antes de analizar determinados aspectos de interés relacionados con las respuestas de los participantes en la prueba.

En primer lugar, era imprescindible plantear claramente los objetivos de la encuesta para evitar errores de especificación (Cea d'Ancona 2004: 36); esto implica, a su vez, definir los conceptos que permitirían analizar el constructo que se investiga (Cea d'Ancona 2004: 192-193). En este caso, por analogía con las investigaciones previas llevadas a cabo, se intentaba comprender la "valoración" de la traducción y de los profesionales de la traducción dentro del sistema literario español (en lengua castellana, por ser la que concentra a mayor número profesionales). Por tanto, siguiendo distintas combinaciones, se recurrió a unos conceptos fáciles de comprender y de uso habitual, como "importancia otorgada", "remuneración", "reconocimiento de la labor profesional", "respeto de los derechos profesionales" y "visibilidad pública".

En segundo lugar, se planteaba la cuestión de la longitud del cuestionario, puesto que de ella iba a depender en buena medida el número de respuestas. Por una parte, ha de tenerse en cuenta que un número importante de los posibles encuestados eran profesionales en régimen de autónomo y, en ocasiones, con una situación laboral precaria (cf. Macías Sistiaga \& Fernández-Cid 2003), que probablemente no querrían dedicar excesivo tiempo a un cuestionario. La tradición en investigación psicosocial avala, por otra parte, un planteamiento basado en la brevedad, ya que, además de afirmar que "la calidad de una escala [...] no puede apreciarse por el número de ítems" (Morales Vallejo 2000: 68), advierte acerca del posible respondent burden (Sharp \& Frankel 1983): el nivel de molestia experimentado por los encuestados cuando el test se hace largo, hecho que puede afectar indirectamente a la 
fiabilidad. Así se puede observar, por ejemplo, que el 62\% de las encuestas llevadas a cabo ente 1964 y 1973 tenían 10 ítems (Bidnick 1975) o que se haya cimentado, con posterioridad, la preferencia por un número de ítems situado entre 20 y 40 (Nunnally 1978: 605). Por último, se tuvo en cuenta también el ya asentado Método de Diseño Total (Total Design Method) de Dillman (2000), cuyos puntos principales sugieren la sencillez del cuestionario, la búsqueda del interés para el encuestado -haciendo referencia a cuestiones que le importen-y la facilidad para responder. De esta manera, se procuró plantear un número de ítems manejable (15), con una mayoría de preguntas cerradas y medidas según la escala Likert, que facilita tanto la respuesta como la corrección. Asimismo, se añadieron dos preguntas más amplias, de tipo abierto, que permitieran recabar otras informaciones variadas, que se relacionaban con la línea de investigación ya iniciada; aunque los datos obtenidos en tales preguntas plantean más dificultades de sistematización, su interés reside en que proporcionan una "apertura cualitativa" (Ortí 1992) que, en ocasiones, puede ofrecer posibilidades de investigación, como se comentará posteriormente.

Por último, se debía proceder a la selección de la muestra que serviría para evaluar el cuestionario piloto; en este proceso, si bien la opinión de los expertos pueda ser útil, es imprescindible recurrir a sujetos que formen parte de la población estudiada (Cea d'Ancona 2004: 37). El número de casos no necesita ser demasiado alto (se tiende a usar grupos inferiores a 100 personas) y la selección, para los propósitos del análisis, tampoco se requiere que sea aleatoria, aunque en este caso se prefirió mantener el carácter aleatorio de la misma. Para ello, se recurrió a los ficheros de las principales asociaciones de traductores de libros (ACEtt, ACEC y ASETRAD); con esa base, se eligió, por orden alfabético, a 200 personas que cumplieran los siguientes criterios: tener el castellano como lengua de llegada (dado que las condiciones de trabajo varían entre las distintas lenguas oficiales), estar especializadas en traducción literaria-humanística y poseer una dirección de correo electrónico de contacto (fórmula cada vez más habitual, aunque no exclusiva). En este punto, resulta importante tener en cuenta la cobertura del marco muestral (Cea d'Ancona 2004: 41): dado que todos los listados disponibles son y serán siempre restrictivos, ha de considerarse en qué medida puede afectar este hecho a la selección de sujetos. En el caso de esta investigación, es importante observar que todos los sujetos del pretest estaban afiliados a alguna asociación de traductores, lo que implica una voluntad profesional o semiprofesional y una conciencia de sus derechos y de la situación laboral en la que se sitúan: al no existir un colegio profesional de traductores, la pertenencia a una asociación no supone una condición previa, sino una elección; por tanto, se 
convierte en una variable importante. Pese a todo, precisamente esa toma de conciencia respecto a su condición laboral podía beneficiar a los propósitos de la investigación, ya que se trabaja con personas cuya opinión tiende a estar formada y asentada. En cualquier caso, con vistas al desarrollo futuro de la encuesta, sería conveniente ampliar el marco muestral mediante otros listados y ficheros, de forma que se evite un posible error de no cobertura (Cea d'Ancona 2004: 105-115).

\subsection{La participación como posible indicador laboral}

Tras el envío de los 200 cuestionarios, se recibieron 84 cuestionarios cumplimentados, aunque sólo 78 de ellos resultaron válidos; asimismo, 10 de los posibles encuestados ( $5 \%$ del total) no recibieron el cuestionario, al ser incorrecta o estar inactiva su dirección de correo. A ello debe sumarse el hecho de que 12 de los encuestados (6\% del total) afirmaran haber abandonado la práctica profesional de la traducción; 9 de ellos se excusaron de esta forma en sus correos de respuesta, mientras que 3 lo indicaron en el cuestionario cumplimentado, razón por la cual tuvieron que ser descartados. El porcentaje de respuesta se sitúa, por tanto, en el 42\% (39\% descontando los no válidos), dato que, si bien no puede considerarse del todo satisfactorio, adquiere más valor por comparación con otras investigaciones previas, como las financiadas por ACEtt en las últimas décadas: la encuesta realizada en 1996 y dirigida sólo a miembros de esta asociación (Macías Sistiaga, Fernández-Cid \& Martín Caño 1997) obtuvo un porcentaje de respuesta en torno al $40 \%$, mientras que la realizada en 2002 por el mismo equipo y con un marco muestral semejante (Macías Sistiaga \& Fernández-Cid 2003) obtuvo una respuesta del 18\%. Conviene detenerse en la explicación que los investigadores contratados por ACEtt sugirieron para este posible descenso en la participación:

La disminución del porcentaje de participación [...] es preocupante tratándose de un grupo caracterizado, como se viene apuntando, por padecer unas condiciones laborales en las que la fragmentación, la incertidumbre, la debilidad de partida en la negociación contractual, se muestran dominantes. La influencia del contexto o marco, en este caso referido al mundo laboral-productivo, ayuda a explicar que estas condiciones, lejos de incitar posiciones más participativas y reivindicativas deriven en manifestaciones de desmotivación y falta de implicación en los asuntos colectivos. (Macías Sistiaga \& Fernández-Cid 2003: 5-6)

Este planteamiento de apatía, unido a las frecuentes protestas públicas de las asociaciones de traductores (cf. Azancot 2003; Rodríguez Marcos 2006; Collera 2007) y al porcentaje ya mencionado de personas -pequeño, pero 
apreciable- que manifestaban haber abandonado la traducción profesional por razones económicas, muestra un ámbito profesional que se conecta claramente con la descripción que Albert Hirschman (1970) hizo de las entidades y sociedades en crisis, donde los actores se mueven entre la opción de "salida" (exit) y la de "voz" (voice), es decir, entre el abandono de la entidad y la denuncia de la situación existente. Ciertamente parece que la interacción de estas actitudes evidencia, en sí misma, una situación "crítica" en lo que concierne al ejercicio profesional de la traducción, ya que, por lo general, sólo un sistema con un tipo de funcionamiento erróneo o descompensado muestra con claridad esas tendencias.

\subsection{Los problemas de respuesta y la formación de conceptos}

Por norma, un cuestionario piloto no se emplea con propósitos estadísticos, sino analíticos (Cea d'Ancona 2004: 37), ya que permite afinar las preguntas empleadas y los conceptos utilizados. En el presente caso, las contribuciones derivadas de este análisis no sólo fueron dispares, sino que evidenciaron algunas cuestiones que podrían ser útiles para formar conceptos de trabajo futuro.

En líneas generales, los encuestados apenas manifestaron problemas con la parte principal del cuestionario, correspondiente a las preguntas cerradas: dos personas consideraron que alguna pregunta concreta necesitaba mayor precisión; tres personas manifestaron que el cuestionario resultaba demasiado general, lo que implicaba dificultad para responder; en contraposición, otras cinco indicaron que la brevedad del cuestionario lo hacía fácil de responder y animaba a hacerlo.

Por el contrario, un número muy alto de encuestados manifestaron algún tipo de dificultad para responder a las dos preguntas abiertas del cuestionario: en la primera, se les pedía que -de acuerdo con su experiencia-indicaran editoriales cuyo trato al traductor fuera positivo o negativo; en la segunda, se les pedía que -nuevamente de acuerdo con su experiencia- mencionasen a críticos literarios que prestaran atención a los libros traducidos que analizaban, frente a aquellos que no lo hiciesen.

La abundancia de comentarios que los encuestados plantearon ante estas preguntas no sólo serviría para refinar los ítems en cuestión, sino que, como se irá detallando en los puntos siguientes, propicia la revisión de ciertos conceptos empleados; desde una perspectiva epistemológica reflexiva, que considera el análisis científico como el paso "desde un conocimiento menos verdadero a un conocimiento más verdadero" (Bourdieu, Chamboredon \& Passeron 1975: 20), esta corrección permitiría incluso la apertura de nuevos aspectos de la investigación: como resume la conocida frase de Bachelard, todo 
conocimiento científico es siempre un conocimiento "aproximado, es decir, rectificado" (Bachelard apud Bourdieu, Chamboredon \& Passeron 1975: 20).

\subsubsection{El trato con las editoriales: "la zona gris"}

La pregunta concerniente a las editoriales muestra una tasa de respuesta muy alta, ya que 68 de los 78 encuestados $(87,18 \%)$ indicaron editoriales que podrían situarse dentro de alguna de las categorías. Sin embargo, 35 encuestados expresaron su dificultad para responder a esta pregunta de manera clara, poniendo de manifiesto una serie de cuestiones relevantes.

En primer lugar, varios sujetos indicaron, de forma directa o indirecta, la imposibilidad de considerar como "positivo" o "negativo" su trato con las editoriales, dado que el conjunto de aspectos relacionados con su actividad laboral (plazos, información, remuneración) variaba considerablemente de una editorial a otra y dentro de la misma editorial; resultaba, en su opinión, habitual que una editorial cumpliese positivamente en una de las cuestiones, pero ofreciera otros aspectos negativos:

[...] el trato de los editores es difícil de valorar en su conjunto, sin precisar si se trata de cumplimiento de sus obligaciones respecto a derechos, información, trato, facilidades, puntualidad en los pagos, etc. (Sujeto 68)

La división entre trato positivo/negativo a los traductores de las editoriales es demasiado tajante. Puede ser positivo en unos aspectos, aunque paguen poco, etc. (Sujeto 51)

Unas editoriales son estupendas, pero la remuneración siempre es escasa, otras son estupendas y la remuneración es adecuada pero jamás informan de las liquidaciones, etc., etc. (Sujeto 76)

Así, el sujeto 47 habla de "editoriales con un positivo en todo menos en remuneración"; el sujeto 38 cita a editoriales que "pagan poco, aunque tratan muy bien", frente a otras que "pagan bien pero tratan mal"; el sujeto 32 indica algunas editoriales "pequeñas" que son positivas en "tiempo, atención...", aunque "no en remuneración"; el sujeto 57 menciona a varias editoriales de trato "negativo" y va precisando las razones de cada elección: "(remuneración, desinformación)", "(remuneración pésima)", "(desinformación)", etc.; lo mismo hace el sujeto 61, que menciona a una editorial con "tarifas irrisorias", aunque "el trato profesional y personal con la editora es bueno". El sujeto 53 proporcionaba una imagen muy representativa de la situación, al afirmar que existe "una gran zona gris" entre lo positivo y lo negativo donde se mueven la mayoría de editoriales literarias. 
Un aspecto relevante que se relaciona con esta dificultad para valorar la relación editorial es, asimismo, la impresión de que las editoriales, en contra de cualquier tipo de deontología, tratan de manera muy diferente a un traductor y a otro, o incluso a un mismo profesional en distintos momentos:

Habría que señalar que la misma editorial puede tratar de manera distinta a traductores distintos (emolumentos, atención...). (Sujeto 36)

Seguramente, habrá editoriales que traten muy bien a un traductor y a la vez traten mal a otro. Esto dificulta el hecho de poder hablar con propiedad de qué editoriales respetan los derechos de los traductores o les dispensan un trato adecuado. El problema reside principalmente en la falta de poder actuar de manera conjunta, y también en que las editoriales son, al fin y al cabo, las que tienen la última palabra. Ante esta situación, los traductores muchas veces capitulan ante situaciones que en algunos casos pueden ser inaceptables. (Sujeto 29)

Las empresas suelen regirse por parámetros variables y una misma editorial puede mostrar dos conductas contradictorias con respecto a un mismo traductor. [...] Llevo muchos años en la profesión y unos cuantos peleando por los derechos de los traductores y no creo que se pueda demonizar ni santificar a nadie. (Sujeto 37)

A menudo, el trato que recibe el traductor por parte de cada editorial depende fundamentalmente de la persona concreta con la que se acuerda cada trabajo. Es habitual que el empleado de turno de la editorial intente conservar su equipo de traducción si cambia de empresa. (Sujeto 40)

En esta perspectiva de incumplimientos y tratos desiguales, sigue habiendo, en cualquier caso, un problema que se manifiesta como fundamental: la remuneración, que siempre resulta inadecuada para la tarea realizada y que impide, por tanto, una valoración más amplia de las editoriales. Por ejemplo, el sujeto 56 señalaba que no podía mencionar casos, porque "debido a la pésima remuneración he llegado a trabajar para muy pocas editoriales comerciales". De manera generalizada, el traductor, como elemento más débil de la negociación salarial, se encuentra con una situación donde las elecciones están muy restringidas:

Para ahondar un poco más en el aspecto de la remuneración, hay que destacar también que las tarifas se estancan durante años, y que los editores no se dignan siquiera añadirles el porcentaje de IPC que se calcula cada año. (Sujeto 9)

Para las editoriales, el traductor suele ser el último mono; es decir, cualquier rebaja en el presupuesto del libro afecta en especial a la traducción [...]. (Sujeto 21) 
Lo que sí veo es que, si uno acepta lo que le ofrecen, las editoriales no mejoran las condiciones con el tiempo. Es el traductor el que tiene que pedir más. (Sujeto 45)

[...] existe una costumbre tan ancestral de no pagar bien al traductor que es muy difícil cambiar un hábito tan arraigado. (Sujeto 38)

Para mí, el problema más grave de la traducción editorial es que ésta constituye una de las pocas áreas laborales en las que es el cliente quien fija el precio, y no el proveedor. Como parece lógico, y como ocurriría en cualquier otro ámbito profesional, cuando es el cliente quien fija el precio, éste lo hace siempre a la baja. (Sujeto 64)

Existe, asimismo, la percepción de que las tarifas han empeorado en términos generales, dado que en las últimas décadas han surgido dos problemas nuevos: el pago por caracteres y la duplicidad de tarifas. La primera de estas cuestiones se ha generalizado entre la mayoría de editoriales y hace referencia a un cambio importante en el sistema de tarificación. Tradicionalmente se pagaba una cantidad por folio; cada folio contiene, aproximadamente, 30 líneas de 70 caracteres cada una, aunque no siempre se llena todo ese espacio (p. ej. textos con diálogos, poemas, etc.). En la actualidad, es habitual que los editores calculen el número total de caracteres del texto y lo dividan entre 2.100, es decir, entre el número de caracteres que contendría un folio "ideal" que estuviese completamente lleno (al que se denomina, técnicamente, "holandesa"). De esta forma, se reduce el pago percibido por traducir textos con espacios, como la poesía, el teatro, las novelas con diálogos frecuentes, los libros de aforismos, etc. De acuerdo con una investigación reciente, este sistema puede reducir los ingresos del traductor cerca de un 20\% (Milla \& Pino 2006):

Observo que en los últimos 10 años la situación profesional del traductor literario ha mejorado en lo que concierne al respeto de sus derechos como autor de su traducción. Sin embargo, otros aspectos han empeorado, como pueden ser la remuneración, debido a la generalización del recuento automático de Word para computar los caracteres de un texto [...] (Sujeto 9)

[...] el traductor "de a pie" ha ido ganando en derechos y en visibilidad: se le conoce algo más y se le respeta algo más. Ahora bien, en mi opinión hay un aspecto en el que estamos peor que hace años: el traductor cobra, de media, menos que antes (y ya es difícil). La estrategia editorial del recuento de caracteres es francamente mezquina. (Sujeto 17)

El gran problema son los pagos. Con el sistema actual de cómputo por plantilla, te acaban pagando algo así como 8 ó 9 euros por página; teniendo en cuenta las dificultades (y las horas) que implican muchas obras, es complicado que te salgan las cuentas [...]. (Sujeto 24) 
Asimismo, se considera como elemento negativo la aparición de una duplicidad de tarifas, que afecta a dos grupos que, por unas razones u otras, no se hallan completamente integrados en el colectivo de traductores: los principiantes y los traductores no profesionales, que compatibilizan la traducción con otros empleos. Por las distintas coyunturas profesionales en que se encuentran ambos grupos y por su desconocimiento de las condiciones laborales, pueden llegar a aceptar tarifas muy inferiores a las habituales, lo que implica, además, un perjuicio para todo el colectivo de trabajadores. Esta tendencia empresarial, consistente en recurrir a los sectores más desfavorecidos del mercado para acordar tarifas inferiores y/o peores condiciones laborales, es una constante dentro de cualquier sector profesional en crisis; se pudo observar, por ejemplo, en abril de 2009 en un ámbito laboral muy distinto, la naval de Sestao, donde los trabajadores convocaron paros como protesta contra la subcontratación de trabajadores inmigrantes con sueldos inferiores, situación que se resolvió con un nuevo convenio que obligó a la empresa al mantenimiento de la igualdad salarial (El País 2009):

Los traductores jóvenes trabajan por tarifas de miseria. Hay un dualismo que no había antes. (Sujeto 25)

[...] existen muchas editoriales pequeñas, de catálogo variado (autoayuda, novela negra, romántica, ciencia ficción...), que pagan cantidades vergonzosas y que se aprovechan de la gente que está comenzando (conozco casos de personas que han cobrado ¡4 euros! por página, un insulto). (Sujeto 34)

[...] las cantidades que he cobrado son muy bajas, totalmente desproporcionadas para el tiempo que me ha llevado cada una de estas traducciones; por lo tanto, si no hubiese tenido otros trabajos mientras tanto, sé que lo habría tenido muy difícil para llegar a fin de mes. En mi caso, la traducción ha sido "un extra" (muy arduo, la verdad). (Sujeto 17)

[...] pocos traductores viven exclusivamente de la traducción, por lo cual, los que tienen otro trabajo no necesitan lo que ganan en la traducción para vivir. (Sujeto 38)

La problemática es muy distinta para el veterano o el novel y, sobre todo, para el "profesional" o para el "académico" (profesor universitario o demás) que completa un sueldo o trabaja por "currículum" o por placer. (Sujeto 54)

Un último problema derivado del trato editorial se asocia con la Ley de Propiedad Intelectual (LPI), aprobada en noviembre de 1987, y con la puesta en vigor del Texto Refundido de la LPI en abril de 1996; de acuerdo con la LPI, el traductor se convierte en autor y propietario de su traducción, lo que implica, entre otras cuestiones (Murillo 2007), que se le concede el cobro por derechos 
de autor y también por cesiones a terceros (reedición de una obra por parte de una editorial distinta de la que pagó la traducción). En la última de las encuestas financiadas por ACEtt (Macías Sistiaga \& Fernández-Cid 2003: 7478), ya se advertía el incumplimiento habitual de la LPI, además de la desinformación en la que las editoriales mantenían a los traductores; esta situación parece mantenerse, de acuerdo con los comentarios de los encuestados:

La mejoría de la situación de los traductores obedece a un solo factor: la LPI. Su aplicación práctica deja mucho que desear y el trato general que reciben los traductores sigue siendo de pena, salvo escasas y honrosas excepciones por ambas partes. (Sujeto 7)

También observo que todavía no es práctica general el que los editores permitan al traductor revisar las pruebas de la traducción, ni tampoco el envío de información sobre liquidaciones de derechos de autor. Tampoco suelen informar los editores de las cesiones de una traducción a otras editoriales. (Sujeto 9)

El respeto a los derechos profesionales ha mejorado mucho en cuanto no afecta a la remuneración, pero por ejemplo los derechos de autor siguen sin percibirse debidamente. Hay reediciones oscuras, de las que uno se entera por Cedro y no por la editorial, o de las que nunca llega a enterarse, cesiones a terceros igualmente oscuras. (Sujeto 25)

Las editoriales siguen redactando contratos que no se ajustan a la LPI. Muchas editoriales no envían liquidaciones anuales. El traductor pierde el control del texto una vez lo ha entregado y no tiene oportunidad de aceptar o rechazar los cambios de correctores o editores. (Sujeto 41)

En suma, el conjunto de precisiones planteado por los encuestados a esta primera pregunta abierta, relativa al trato con las editoriales, define un panorama complejo, confuso y, en general, negativo, donde ni siquiera los propios participantes saben exactamente a qué atenerse; su opinión no puede ser global, en la medida en que forman parte de un mercado fragmentado, inestable y con un marcado desequilibrio. Su situación laboral, por otra parte, no se asemeja a la habitual en las profesiones que podrían denominarse "liberales" o "intelectuales", sino que se acerca más a la de otros trabajadores no cualificados; una reciente encuesta de CEATL a nivel europeo (Fock, de Haan \& Lhotová 2009: 69) señala que los ingresos brutos de una gran parte de los traductores literarios se sitúan por debajo de los percibidos por los obreros industriales, no llegando, en diversos países, a igualar siquiera los dos tercios de un salario industrial. El traductor, en suma, se define como un "trabajador proletarizado", puesto que el "rasgo específico que distingue a los traductores en la actualidad [...] es su condición individual de prescindibles, intercambiables" 
(Martínez-Lage \& Sánchez Lizarralde 1997: 13); lo que, por otra parte, no deja de enlazarse con un mercado mundial, más amplio, donde se insiste en la "contingencia" del trabajador y en la necesidad -casi la obligación- de que sea adaptable a las condiciones fluctuantes (Sennett 1998; Bauman 2005). De todo ello se colige, por tanto, la necesidad de que cualquier investigación en este campo tenga en cuenta ese carácter variable, confuso de la situación laboral del traductor literario y la considerable dificultad de estos profesionales para establecer sus parámetros de orientación en el mercado.

\subsubsection{La función de la crítica y la conciencia del sistema literario}

La segunda de las preguntas abiertas que se incluía en el cuestionario se refería a los críticos literarios y reseñistas; se solicitaba a los encuestados que mencionaran a aquellos que, de acuerdo con su experiencia, concediesen (o no) importancia a la traducción que analizaban. Nuevamente, las respuestas evidencian dificultades en torno al concepto planteado, aunque su carácter sea distinto al que se observaba en la primera de las preguntas abiertas.

En primer lugar, se observa un porcentaje de respuesta relativamente bajo, ya que sólo 34 de los 78 encuestados (43,59\%) contestaron. El dato de interés aparece cuando se procede a un desglose por categorías, que indica una diferencia peculiar: entre los encuestados que afirmaban tener la traducción como actividad principal, la tasa de respuesta se reduce hasta volverse poco relevante, pues sólo 14 de los 54 participantes (25,93\%) respondieron; por el contrario, en el colectivo, bastante menor, de encuestados que compatibilizaban la traducción con otras actividades profesionales, la tasa aumentaba de manera muy marcada, dado que 20 de los 24 sujetos (83,33\%) contestaron. Esta diferencia, aunque sea imprescindible considerarla como provisional por el carácter probatorio del cuestionario, sugiere una posibilidad de trabajo importante: una conciencia muy distinta entre profesionales y no profesionales en lo que concierne a la crítica literaria y su importancia dentro del sistema literario. En líneas generales, los profesionales que se pronuncian en torno a la cuestión suelen apuntar al desconocimiento o desinterés de los críticos, es decir, ponen en duda la propia conveniencia y posibilidad técnica de que una traducción sea juzgada por aquellos:

[...]se trata de un terreno para el cual no está preparado técnicamente; la mayoría de la crítica confunde hallazgos o defectos del autor con hallazgos o defectos del traductor, y viceversa. (Sujeto 37)

[...]la mayoría de críticos sólo mencionan el nombre del traductor pero no hacen crítica de la traducción, y cuando dicen algo de ésta suele ser negativo, raras veces positivo. (Sujeto 39) 
[...] el juicio sobre la calidad suele resumirse, las menos de las veces, en unas pocas líneas, en la mayoría de casos, con un par de adjetivos. (Sujeto 47)

Las menciones a los traductores suelen ser breves y generales, cuando existen, del tipo "por cierto, la traducción no está mal/es muy mala". No suele hacerse un análisis riguroso ni con conocimiento de causa. (Sujeto 53)

La pregunta es improcedente. 1. la inmensa mayoría [de los críticos] carece de un conocimiento suficiente de la lengua de origen. 2. y caso de tenerlo es prácticamente imposible que haya podido confrontar completamente ambos textos. (Sujeto 55)

No he leído nunca un comentario inteligente de un crítico en esta materia, sin duda porque desconocen los originales. (Sujeto 59)

Una segunda cuestión en la que coinciden las respuestas de los sujetos -con las matizaciones ya hechas acerca de la amplia diferencia en número- es la relación entre los críticos literarios y la visibilidad que pueden otorgar a una traducción. Aun así, puede apreciarse, de nuevo, cierta disparidad: mientras los profesionales que aluden a este problema señalan la importancia que la visibilidad de los traductores podría tener para la mejora de su situación laboral, los encuestados no profesionales hacen más hincapié en la tarea del crítico como mediador $u$ orientador de la lectura, es decir, como agente que influye en la valoración de la obra traducida:

En mi opinión, el problema de la traducción es [...] el desconocimiento del gran público, que no considera que el traductor sea un profesional cuyo trabajo deba ser reconocido y bien remunerado, cosa que favorece a los editores [...]. Está, además, y por todo lo anterior, el escaso reconocimiento del trabajo del traductor, no sólo por los medios de comunicación (que sólo hablan de la traducción cuando es mala) sino también por los editores (que muchas veces ni siquiera ponen su nombre en los catálogos). (Sujeto 38)

[...] pese a la labor tenaz y constante de la asociación de traductores literarios, ACEtt, por mejorar la visibilidad del traductor en la sociedad, todavía no se han alcanzado buenos resultados, notablemente en la prensa cultural, donde los críticos literarios siguen omitiendo el nombre del traductor en sus reseñas de libros y, desde luego, muy rara vez alaban la labor del traductor. Para denostarla no tienen en cambio tantos reparos. (Sujeto 9)

En los últimos 10 años ha mejorado ligeramente la situación del traductor de libros en cuanto a visibilidad y derechos, no así en cuanto a remuneración y reconocimiento público. Es verdad que nuestra lucha ha dado y sigue dando algunos frutos, pero seguimos encontrándonos con el muro infranqueable de la crítica literaria y los medios de este país que siguen sin reconocer la labor de los traductores en favor de la difusión del libro. (Sujeto 12) 
Pocos críticos se detienen en la importancia y/o calidad de la traducción. Generalmente se trata de expertos en el tema que reseñan. La mayoría ignoran al traductor y la traducción, sobre todo, los "figuras" habituales en la sección de Libros que creen "saber" de todo y, encima, condicionan la demanda de la lectura. (Sujeto 22)

La atención a la traducción literaria en la crítica literaria está estrechamente vinculada a la propia actividad como traductor del crítico. Se menciona, acaso, cuando se trata de traductores consagrados o de nuevas versiones de clásicos (Proust, Flaubert, Dickens, etc.) (Sujeto 10)

La ignorancia de los críticos literarios -que, cada vez con más frecuencia, suelen ser periodistas reciclados- en torno a las lenguas y literaturas extranjeras les impide comprender el esfuerzo que supone una traducción y, sobre todo, la importancia de una buena traducción. Una literatura depende también de las traducciones. (Sujeto 24)

Todo el engranaje editorial en España está enfocado tan sólo para tener unos rendimientos económicos. Y aquí se supeditan todas las partes, desde las editoriales hasta los lectores. Los críticos, que tendrían que hacer de puente entre ambas partes, actúan de manera contraria y tan sólo contribuyen a agravar la situación. Al final, el traductor en España se ha acostumbrado a pensar que si en una reseña no hablan mal de su traducción, la reseña es buena. El hecho de no verse mencionados es un aspecto positivo, lo cual indica mucho de la propia visión que se tiene en España. (Sujeto 29)

Tanto editores como críticos están sujetos a los vaivenes de la industria y se rinden antes al mercado que a criterios éticos y estéticos propios. (Sujeto 37)

Pese al carácter forzosamente provisional de estas distinciones entre grupos, cabe plantearse con un mínimo de certeza que las disparidades planteadas por esta segunda pregunta sugieren precisiones útiles en la investigación: por un lado, parece percibirse un cierto desinterés del traductor profesional ante la tarea del crítico literario, que se considera, en todo caso, de calidad deficiente; por otro, se advierte con claridad un mayor interés hacia esta tarea por parte de los traductores no profesionales, quienes parecen considerar relevante su tarea dentro del sistema literario (no tanto por una cuestión de calidad como por una cuestión de importancia dentro del sistema de legitimación literario). En la medida en que la crítica constituye una de las principales "formas de dominación literaria" (Casanova 1999: 172) y la traducción es la gran "institución de consagración" literaria (Casanova 1999: 198), estas diferencias en sus zonas de interacción podrían servir como marco para una perspectiva útil de trabajo. 


\section{Conclusiones: el acercamiento dialéctico y las posibilidades de investigación}

En su correo electrónico de respuesta, uno de los encuestados incluía una frase que podría definir la necesidad de una forma de trabajo basada en la interacción: "No se da cuenta uno de lo triste que está el sector hasta que lo piensa". Este "emerger" de una conciencia diferente respecto a la propia labor alude perfectamente al propósito de investigación: a la vez que el cuestionario interroga a los sujetos y les plantea posibles dudas sobre su actuación, el análisis posterior de estas reacciones sirve igualmente para poner en duda el planteamiento propio del investigador, que se resituará respecto a su objeto de trabajo.

Los problemas planteados por el cuestionario piloto tratado en este artículo ponen de relieve la necesidad de una "vía de comunicación" entre el investigador y los sujetos del campo analizado. Las dos preguntas abiertas que se han analizado podrían considerarse, en un primer momento, simplemente mal formuladas o problemáticas en lo que concierne a la evaluación del cuestionario, ya que ambas presentan, de formas distintas, complicaciones para los encuestados. Sin embargo, desde la perspectiva constructivista y dialéctica, estas necesidades de definición suponen un marco donde plantear interrogantes que ayuden a la investigación. El propósito inicial de estas preguntas era hallar datos: individuos y empresas que correspondieran a unos parámetros de trabajo; sin embargo, los cuestionarios no satisficieron esa primera intención, sino que mostraron algo distinto: una situación, un ámbito más complejo que conlleva unas necesidades de trabajo diferentes a las que se plantearon inicialmente. No se obtuvo, como se pretendía en un principio, un listado más o menos preciso de editoriales de trato "positivo" o "negativo", ni un conjunto de críticos con diversas actitudes ante la literatura traducida. Sin embargo, se hallaron otras intuiciones que permiten una forma diferente de trabajo. Aunque en todo momento conviene recordar el carácter provisional y no extrapolable de un cuestionario piloto, la abundancia de datos obtenidos, puestos en relación con otras investigaciones previas, permiten definir algunos conceptos útiles para plantear la investigación futura: en primer lugar, se observa una conciencia por parte de los encuestados de hallarse en un sector, el editorial, caracterizado por la confusión, la indefinición y la multiplicidad de tratos, lo que implica una mayor dificultad de orientación personal; por otro, se percibe una cierta impresión polarizada del sector, sobre todo en torno a los ejes novato-veterano y profesional-no profesional; por último, parece plausible considerar una diferencia de actitud entre subgrupos en lo que respecta a la crítica literaria, el sistema literario y la idea de visibilidad. Estos 
rasgos parecen aludir, en suma, a un ámbito laboral marcado por la individualización y la sectorización, es decir, un ámbito donde los trabajadores poseen pocos marcos de referencia más allá de los personales y donde las identidades grupales no se forman tanto por la voluntad-actividad conjunta de los miembros, sino por sus condiciones laborales impuestas.

Esta percepción coincide con la desarrollada por otros investigadores, que han señalado la dificultad del sector de la traducción para definirse como profesión a causa de "una socialización deficiente de los profesionales" y de "una identidad común poco definida" (Monzó 2006: 173); la ambigüedad llega hasta el punto de que se plantee la conveniencia de hablar acerca de varias profesiones de traductor, en la medida en que los traductores de un ámbito o categoría suelen desconocer las condiciones en que se hallan los de otras (Gouadec 2007: xiii-xiv). Asimismo, estas dificultades de delimitación enlazan con la preocupación expresada por algunos encuestados $\left(n^{\circ} 7,9,22\right.$, 26) en torno al intrusismo laboral y la necesidad del asociacionismo, cuestión especialmente compleja, no sólo por la división que causa la posibilidad de regular el acceso profesional a la traducción mediante algún tipo de normas o requisitos (Gouadec 2007: 252-257), sino también porque, en el momento actual, la Directiva Europea de Servicios recomienda a los gobiernos europeos la supresión o flexibilización de la colegiación obligatoria que exige el ejercicio de numerosas profesiones liberales (Editorial 2008). Más allá del carácter ambiguo de la propuesta, lo que evidencia esta inquietud es, nuevamente, la dificultad del colectivo laboral para definirse y, a la vez, la voluntad de una parte para alcanzar alguna clase de identidad colectiva mediante órganos de representación.

Al hilo de esta indefinición, conviene señalar una precisión pertinente de Žižek (1989: 230-232) como crítica a Adorno (1970) y que remite una vez más al problema de los paired concepts que se señaló al inicio. De acuerdo con Adorno, en la actualidad no se puede formular una definición adecuada de la "Sociedad", puesto que, cuando se intenta hacerlo, aparecen una serie de determinaciones opuestas, que se excluyen mutuamente: por una parte, la sociedad como un todo orgánico que abarca a los individuos ("organicismo"); por otra, la sociedad como un vínculo entre individuos atomizados ("individualismo"). Sin embargo, en un acercamiento dialéctico, esta contradicción funciona precisamente como respuesta: el antagonismo que revela el concepto de Sociedad funciona como su definición misma -es decir, el objeto de estudio se define justo a través de la característica que parecía impedir el acceso a él-. Las discrepancias, por tanto, que se plantearon a raíz del cuestionario piloto son, al final, las que evidencian el carácter peculiar del grupo analizado: 
la dificultad que se encuentra cuando se intenta definir el colectivo de los traductores literarios no sólo constituye una de sus características principales en tanto que objeto de estudio, sino que pone de relieve una vez más el carácter borroso de las categorías humanas ( $c f$. Wittgenstein 1953: 66-71; Rosch 1978; Lakoff 1987) y la vigilancia epistemológica que conviene aplicarles en cualquier investigación social.

Estas precisiones permiten ver de un modo distinto la utilidad de las vías de comunicación entre observador y actores; no se trata tan sólo de una cuestión de datos o conocimientos, sino, sobre todo, de un reajuste de perspectivas y, por tanto, de la posibilidad de construir el objeto sociológico de una manera más precisa. Esta preocupación remite, de nuevo, al concepto de reflexividad y, en especial, a la precaución imprescindible frente al "sesgo escolástico" (Bourdieu 2004: 22), es decir, ante el riesgo de que el investigador, en su acercamiento a los sujetos, acabe por plantear "preguntas de investigador", preguntas que sólo pueden formularse desde fuera y que, por tanto, dificultarán la comunicación con los actores; en el caso del cuestionario piloto que se ha analizado, podría plantearse que las preguntas abiertas, al funcionar en cierto modo como "preguntas de investigador", obligaron a los encuestados a proponer respuestas diferentes a las esperadas, de marco más amplio, y reveladoras por tanto de unas tensiones que no estaban previstas en las preguntas iniciales. De ahí, en suma, que este sistema de trabajo constructivista, dialéctico, sólo pueda plantearse a partir de una defensa del proceso investigador como "error rectificado" (Bachelard) y desde la conciencia de la utilidad del "falso reconocimiento" (el primer error de identificación permite acceder de forma distinta al objeto estudiado y superar convencimientos propios; cf. Žižek 1989: 97-100).

Por último, cabe apuntar que algunas de las características antagónicas evidenciadas por el cuestionario analizado permiten analizar de una forma más concreta uno de los principales conceptos planteados en la introducción: la "brecha de paralaje" (Žižek 2005). Los planteamientos empíricos confirman el interés que podría tener un acercamiento a la traducción literaria que, en lugar de limitarse a una única perspectiva del ámbito, tenga en cuenta las múltiples oposiciones (paralajes) que conforman el ámbito de trabajo y que, por tanto, tienen presencias variables en sus distintos aspectos: la traducción literaria como elemento cultural indispensable para la literatura universal y, al mismo tiempo, sometido de forma irremediable a cuestiones geopolíticas (Heilbron 1999) y económicas (Fernández 2007a); generadora de prestigio para los autores y, sin embargo, mantenida por tradición como actividad subalterna; fundamental en términos de mercado editorial, pero sustentada en 
una profesión insegura y proletarizada. El reconocimiento de ese intersticio formado por el conjunto de paralajes parece un punto de partida idóneo para la investigación, siguiendo un planteamiento sociológico que, en la construcción de su objeto de estudio, logre evitar en la medida de lo posible los efectos limitadores de las oposiciones conceptuales.

\section{Bibliografía}

ADORNO, Theodor Wiesengrund. (1970) "Society". Salmagundi, 10-11. pp. 144-153.

AzAnCot, Nuria. (2003) "Traductores. El estado de la cuestión". El Cultural, 30 de octubre de 2003. pp. 8-9.

Bauman, Zygmunt. (2005) Liquid Life. Cambridge: Polity Press.

BENDIX, Reinhard \& Bennett Berger. (1959) "Images of Society and Problems of Concept Formation in Sociology". En: Gross, Llewellyn. (ed.) 1959. Symposium on Sociological Theory. Nueva York: Harper and Row. pp. 92-118.

BiDniCK, Marilyn. (1975) The Methodology of Measurement: An Analysis of Selected Scaling Techniques and Their Applications in Sociology. Michigan: Xerox University Microfilms.

BOURDieu, Pierre. (2001) Science de la science et réflexivité. París: Éditions Raisons d'agir.

Bourdieu, Pierre. (2004) "Lobjectivation du sujet de l'objectivation". En: Heilbron, Johan; Remi Lenoir \& Gisèle Sapiro (eds.) 2004. Pour une histoire des sciences sociales. París: Fayard. pp. 19-23.

Bourdieu, Pierre; Jean-Claude Chamboredon \& Jean-Claude Passeron. (1975). Le métier de sociologue. París: Mouton. Citado por la traducción de Fernando Hugo Azcurra y José Sazbón: El oficio de sociólogo. Presupuestos epistemológicos. Madrid: Siglo XXI Editores, 2005.

CaSAnova, Pascale. (1999) La république mondiale des lettres. París: Éditions de Seuil. Citado por la segunda edición revisada (2008).

CEa D'AnCona, María Ángeles. (2004) Métodos de encuesta. Teoría y práctica, errores y mejora. Madrid: Síntesis.

Collera, Virginia. (2007) "Traducciones crecientes, dinero menguante". El País, 6 de enero de 2007. pp. 39.

CorCufF, Philippe. (1995) Les nouvelles sociologies. París: Nathan. Citado por la traducción de Belén Urrutia, Las nuevas sociologías. Construcciones de la realidad social. Madrid: Alianza Editorial, 2005.

Dillman, Donald Andrew. (2000) Mail and Internet surveys: the tailored design method. Nueva York: Wiley. 
DURKHEIM, Émile. (1897) "La conception matérialiste de l'histoire". En: Durkheim, Émile. (1970) La science sociale et l'action. Paris: Presses Universitaires de France, pp. 245-254

Editorial. (2008) "Profesiones no tan libres". El País, 5 de octubre de 2008, p. 36.

FERNÁNDEZ, Fruela. (2007a) "La tradición alemana en Haroldo de Campos: omisiones, tendencias y la vieja postmodernidad”. En: Santana, Belén; Silvia Roiss $\& \mathrm{M}^{a}$ Ángeles Recio (eds.) 2007. Puente entre dos mundos: últimas tendencias en la investigación traductológica alemán-español. Salamanca: Ediciones Universidad de Salamanca. pp. 113-122.

FERNÁNDEZ, Fruela. (2007b) Suplementos culturales, crítica literaria y literatura traducida en España: una propuesta de investigación. Proyecto de Investigación Tutelada (inédito). Universidad de Granada.

FERNÁNDEZ, Fruela. (2008) "Reivindicación y desconfianza, o algo más sobre las (malas) relaciones (necesarias) entre crítica y traducción". Vasos comunicantes 38, pp. 55-68.

FERNÁNDEZ, Fruela. (en preparación) Suplementos culturales, literatura traducida y recepción crítica en España (1999-2008). Tesis doctoral. Universidad de Granada.

Fock, Holger; Martin de Haan \& Alena Lhotová. (2009) Comparative income of literary translators in Europe. Versión electrónica: <http://www.ceatl.eu/docs/ surveyuk.pdf>

FOUCAUlt, Michel. (1972) "Les intellectuels et le pouvoir. Entretien avec Gilles Deleuze”. L'Arc 49. pp.3-10. Citado según la edición de Miguel Morey: Un diálogo sobre el poder y otras conversaciones. Madrid: Alianza Editorial, 2007.

GouAdec, Daniel. (2007) Translation as Profession. Amsterdam \& Philadelphia: John Benjamins.

HARRIS, Marvin. (1964) The nature of cultural things. Nueva York: Random House.

HARRIS, Marvin. (1979) Cultural materialism: The struggle for a science of culture. Nueva York. Vintage.

Headland, Thomas N.; Kenneth L. Pike \& Marvin Harris. (1990) Emics and Etics. The Insider/Outsider Debate. Newbury Park \& Londres: Sage Publications.

HeILBRON, Johan. (1999) "Towards a Sociology of Translation. Book Translations as a Cultural World System", European Journal of Social Theory 2:4. pp. 429-444.

HiRSCHMAn, Albert. (1970) Exit, Voice and Loyalty. Responses to Decline in Firms, Organizations and States. Cambridge (EEUU) \& Londres: Harvard University Press.

Karatani, Kojin. (2001) Transcritique. Hihyōkūkansha: Tokyo. Citado según la traducción de Sabu Kohso: Transcritique: on Kant and Marx. Cambridge: MIT Press, 2003. 
LAKOFF, George. (1987) Women, Fire and Dangerous Things. Chicago: Chicago University Press.

Macías Sistiaga, Carmen \& Matilde Fernández-Cid. (2003) Informe sobre la situación del traductor de libros en España. Versión electrónica: <http://www. acett.org>.

Macías Sistiaga, Carmen; Matilde Fernández-Cid \& Ángel Martín Caño. (1997) "Encuesta a traductores. Informe de resultados". En: ACE-Traductores (eds.) 1997. Libro Blanco de la Traducción en España. Madrid: ACE-Traductores. pp. 25-95.

MarTínez-Lage, Miguel \& Ramón Sánchez Lizarralde (1997): "Presentación". En: ACE-Traductores (eds.) 1997. Libro Blanco de la Traducción en España. Madrid: ACE-Traductores, pp. 9-23.

Milla, Carlos \& Marta Pino. (2006) "De te fabula narratur: los sistemas de cómputo y el rendimiento del trabajo de traductor en el sector editorial". Vasos Comunicantes 34. pp. 35-64.

Monzó, Esther. (2006) "¿Somos profesionales? Bases para una sociología de las profesiones aplicada a la traducción”. En: Parada, Arturo \& Oscar Diaz Fouces (eds.) 2006. Sociology of Translation. Vigo: Universidade de Vigo. pp. 157-176.

Morales Vallejo, Pedro. (2000) Medición de actitudes en psicología y educación. $2^{a}$ edición revisada. Madrid: Universidad Pontificia de Comillas.

Murillo, Enrique. (2007) "Otro coste editorial". El País, 6 de enero de 2007. pp. 39.

Nunnally, Jum. (1978) Psychometric Theory. Nueva York: McGraw-Hill.

ORTí, Alfonso. (1992) "La apertura cualitativa y el enfoque estructural". En: García Ferrando, Manuel; Jesús Ibáñez \& Francisco Alvira (eds.) 1992. El análisis de la realidad social: métodos y técnicas de investigación social, Madrid: Alianza Universidad. pp. 189-221.

PAís, El. (2009) "El astillero de Sestao protesta por la contratación de extranjeros". El País, 25 de abril de 2009. p. 27.

PIKE, Kenneth L. (1954) [1967] Language in relation to a unified theory of the structure of human behaviour. La Haya: Mouton.

Rodríguez Marcos, Javier. (2006) "Homero no escribía en español". Babelia, 28 de octubre de 2006. pp. 2-3.

RosCH, Eleanor. (1978) "Principles of categorization". En: Rosch, Eleanor \& Barbara Lloyd (eds.) 1978. Cognition and categorization. Hillsdale: Erlbaum Associates. pp. 27-48.

Rubinstein, David. (1981) Marx and Wittgenstein. Londres: Routledge \& Keegan.

SennetT, Richard. (1998) The Corrosion of Character. The Personal Consequences of Work in the New Capitalism. Nueva York \& Londres: W.W. Norton \& Company. 
SHARP, Laure \& Joanne Frankel. (1983) "Respondent Burden: A Test of Some Common Assumptions". Public Opinion Quarterly 47. pp. 36-53.

Wittgenstein, Ludwig. (1953) Philosophische Untersuchungen. Oxford: Basil Blackwell. Citado según la edición crítica de Joachim Schulte: 2001. Wissenschaftliche Buchgesellschaft: Frankfurt am Main.

ŽIŽEK, Slavoj. (1989) The sublime object of ideology. Londres: Verso. Citado según la traducción de Isabel Vericat Núñez: El sublime objeto de la ideología. Madrid \& Buenos Aires: Siglo XXI Editores, 2007.

ŽIŽEK, Slavoj. (2005) The Parallax View. Cambridge: MIT Press. Citado según la traducción de Marcos Mayer: Visión de paralaje. Buenos Aires: Fondo de Cultura Económica, 2006. 